\section{Ethnicity, heart failure and the prevention continuum: time to act}

\author{
Amitava Banerjee
}

\section{ETHNICITY, RACE AND CARDIOVASCULAR DISEASE (CVD) IN SOUTH ASIANS}

Every UK Census since 1841 has collected data regarding country of birth and nationality. However, ethnicity was recorded from only the 1991 census onwards. The number of categories and their complexity has increased over the years. Race and ethnicity are overlapping concepts, but 'the term race should be used with caution for its history is one of misuse and injustice'. ${ }^{1}$ Race refers to a person's skin colour and origins, whether by nationality or country of birth. Ethnicity tends to refer more subjectively to a shared history and culture, language, religion and traditions. South Asians are the largest ethnic minority group in the UK (4.9\% in the UK 2011 Census) and provide an important lens for research and action to address ethnic disparities in health and healthcare.

Since the 1980s, inequalities in diagnosis, treatment and monitoring of coronary artery disease (CAD) in ethnic minority communities, particularly South Asians, have been reported. The demonstration that South Asians had higher incidence of CAD (hazard ratio, (HR) 1.35, 95\% CI 1.30 to 1.40 ) but better prognosis (HR $0.78,95 \%$ CI 0.74 to 0.82 ) compared with Caucasians suggested that 'public health initiatives to reduce inequalities in mortality between South Asian and white populations should focus on primary prevention'.2 Further analyses implicate a combination of underlying biological factors as causes (eg, adipose tissue distribution and metabolism), as well as environmental, demographic, social and behavioural factors. Although similar research may guide aetiological, preventive and therapeutic insights in both individuals and populations, other CVDs and non-communicable diseases have not been so well studied.

\section{ETHNICITY AND IMPLANTABLE CARDIOVERTER DEFIBRILLATOR (ICD) THERAPY}

Research and management of heart failure (HF), a major global burden of disease with variations in diagnosis and

Correspondence to Dr Amitava Banerjee, Institute of Health Informatics, University College London, London NW1 2DA, UK; ami.banerjee@ucl.ac.uk treatment within and across countries, can benefit from analyses by ethnicity. ICD therapy is a major component of treatment of both ischaemic and nonischaemic HF, as well as management of ventricular arrhythmias. In their Heart paper, Mistry and colleagues report on ICD rates, regardless of indication, in South Asians, compared with Caucasians in a Leicestershire population of 980328 (of which $15.9 \%$ is South Asian). They conclusively show that ICD implantation rates were lower in South Asians (only $8.1 \%$ of all the procedures performed). South Asians were half as likely to receive ICD therapy, compared with Caucasians (relative risk, (RR) 0.43, $95 \%$ CI 0.37 to $0.49, \mathrm{p}<0.001)$ even when standardised for age (RR 0.75, $95 \%$ CI 0.74 to $0.75, \mathrm{p}<0.001)$. This inequality was most pronounced for secondary prevention ICD implantation (age-standardised RR 0.49 , 95\% CI 0.48 to $0.50, \mathrm{p}<0.001$ ) but was also observed

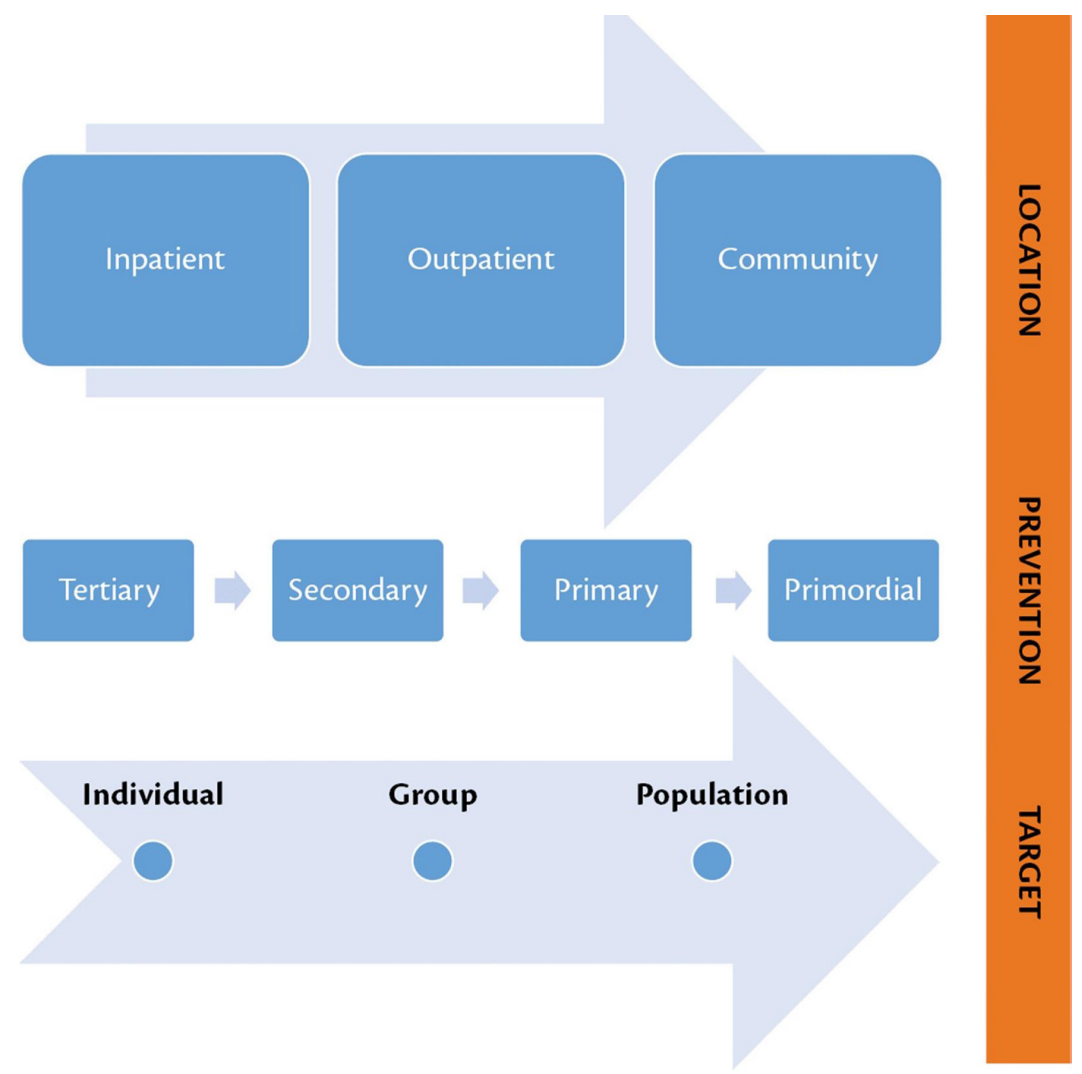

Figure 1 Conceptual framework for prevention in healthcare (adapted with permission from Banerjee $^{7}$ ). clearly relevant. ${ }^{6}$

\section{ETHNIC DISPARITIES IN HF ACROSS THE PREVENTION CONTINUUM}

for the primary prevention indication (age-standardised RR 0.91, 95\% CI 0.90 to $0.91, \mathrm{p}<0.001){ }^{3}$

The majority of published literature regarding ethnic disparities in HF originates from the USA, pertaining to the African-American population. Retrospective analyses in US data suggest that the intersection of gender and race still results in persistent inequalities in ICD implantation. ${ }^{4}$ For example, the Get With The Guidelines-Heart Failure programme found that black (adjusted OR $0.70,95 \%$ CI 0.56 to 0.88 ) and Hispanic patients (adjusted OR 0.68, 95\% CI 0.46 to 1.01 ) were less likely to receive an ICD than their Caucasian counterparts ${ }^{5}$ and that they were over-represented in the $80 \%$ of patients with HF who are not counselled for ICD therapy. Disparities are also present in cardiac resynchronisation therapy (CRT), and socioeconomic factors such as medical insurance are

Mistry and colleagues note, 'Variation in implantation amongst different ethnicities may be in part be due to genetic differences in susceptibility to cardiovascular 
disease, due to socioeconomic factors such as access to healthcare insurance as seen in the USA, or due to unconscious bias from physicians'. ${ }^{3}$ All three potential explanations warrant further investigation. However, in order to understand variation in ICD implantation, we need a much broader understanding of ethnic disparities for HF since the UK context, including South Asians, has been neglected across the whole prevention continuum ${ }^{7}$ (figure 1).

Primordial prevention involves prevention of risk factors themselves. Across world regions, risk factors for HF vary greatly, with CAD most prevalent in Europe and North America, hypertension prevalence highest in Eastern and Central Europe and sub-Saharan Africa, and cardiomyopathy and rheumatic heart disease most common in Latin America, the Caribbean and sub-Saharan Africa, ${ }^{8}$ and therefore primordial prevention strategies for HF will vary greatly by region and ethnicity. High rates of diabetes and hypertension in South Asian communities at country or community level suggest that prevention might vary based on the ethnic diversity of the target population, necessitating context-specific research in ethnic minorities. In the Leicestershire study, the young age of South Asians undergoing ICD implant signals the need for greater primordial prevention. There is a complicated interplay between ethnicity and other social determinants of health, such as socioeconomic status and demographic factors. The major causes of inequalities in health, and even more of inequalities in access to healthcare, are inequalities in wealth, and the majority of the associations seen with ethnicity may be explained, at least in part, by other socioeconomic factors.

Primary prevention is prevention of disease before its biological onset. South Asian ethnicity is associated with a higher prevalence of hypertension, diabetes and $\mathrm{CAD}$, and a lower prevalence of atrial fibrillation than other ethnicities, whether in South Asia or in Western populations. Mistry et al show that rates of CAD were significantly higher in South Asians (69.2\%) than Caucasians (60.1\%, $\mathrm{p}=0.009)$. The higher prevalence of certain risk factors and earlier onset of HF in South Asians (and probably other communities) again implies a tailored approach to primary prevention, in these cases, focusing on hypertension, diabetes and obesity. ${ }^{3}$ However, intervention studies to date are lacking. ${ }^{7}$

Secondary prevention identifies and treats existing disease early before disease symptoms are obvious in order to reduce morbidity and mortality. It can involve treatment of individuals with single or multiple risk factors and/ or early-stage disease, and should be adapted to the burden of disease and outcomes, depending on the region or the ethnic distribution in the population. Consideration of ICDs has to take into account the wider secondary prevention context, including adherence to secondary prevention drugs, access and use to cardiac rehabilitation services and cultural differences in presentation to acute healthcare services. Moreover, whether ICD or CRT, criteria for HF therapy, are based predominantly on white populations, and ethnic minorities are under-represented in trials. Clinical trials of treatments still under-recruit and under-represent ethnic minorities, and there is little evidence to date of coordinated strategies to address these disparities. It is possible that ethnicityspecific QRS duration cut-offs in criteria for device therapy are required, ${ }^{7}$ and response to therapy may also be different in different ethnic groups. The rapid acceleration in research of the genetic basis of HF and other complex chronic conditions has not been accompanied by growth in funding or research in individuals of non-European ancestry, who shoulder a far greater global burden of HF and other NCDs. Without this research, applicability of genetic findings to non-Caucasian individuals is questionable and the biological basis of ethnic variations in HF is more difficult to untangle.

Tertiary prevention is concerned with established, symptomatic disease to prevent further disease progression, morbidity and mortality. In the context of $\mathrm{HF}$, it usually refers to advanced disease, where symptom management is the priority, and is often poorly managed in patients with HF of all ethnicities. South Asians in Leicestershire had more severe HF when presenting for ICD implantation (both in terms of symptoms and left ventricular impairment) than their Caucasian counterparts. In combination with evidence that availability and use of management options in advanced HF, including palliative care, are lower in ethnic minorities, the tertiary prevention gap is clear.

\section{BIG DATA, BIG PICTURE}

Every stage of management of HF from primordial prevention through to tertiary prevention exhibits variation by ethnicity.
Many of these differences are likely to be explained by differences in socioeconomic or risk factor profile (or both of these) rather than the genetic differences alone. Large-scale electronic health records, administrative data and appropriate data linkage, along with consistent and applicable definitions of ethnic groups, introduce the possibility of routine analysis of variation in health and healthcare (including procedures such as ICD implementation) by ethnicity. Although there are substantial gaps in descriptive and intervention research, the holistic consideration of the prevention continuum can help in addressing health disparities by ethnicity.

\section{Twitter Amitava Banerjee @amibanerjee1}

Contributors $A B$ is the sole author of the article.

Funding $A B$ has received grant funding from the BigData@Heart Consortium, under the Innovative Medicines Initiative-2 (116074, supported by the European Union's Horizon 2020 programme) and European Federation of Pharmaceutical Industries and Associations, EFPIA (chair persons DE Grobbee, SD Anker), National Institute for Health Research and the British Medical Association.

Competing interests $A B$ has been an advisory board member for Boehringher Ingelheim, Novo-Nordisk, Astra-Zeneca and Pfizer, all unrelated to this work.

Patient consent for publication Not required.

Provenance and peer review Commissioned; internally peer reviewed.

(C) Author(s) (or their employer(s)) 2020. No commercial re-use. See rights and permissions. Published by BMJ.

\section{Check for updates}

To cite Banerjee A. Heart 2020;106:631-633.

Published Online First 6 February 2020

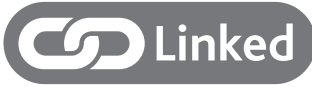

- http://dx.doi.org/10.1136/heartjnl-2019-315978

Heart 2020;106:631-633.

doi:10.1136/heartjnl-2019-316293

\section{ORCID iD}

Amitava Banerjee http://orcid.org/0000-0001-87413411

\section{REFERENCES}

1 Bhopal R. Glossary of terms relating to ethnicity and race: for reflection and debate. J Epidemiol Community Health 2004;58:441-5.

2 Zaman MJS, Philipson P, Chen R, et al. South Asians and coronary disease: is there discordance between effects on incidence and prognosis? Heart 2013;99:729-36.

3 Mistry A, Vali Z, Sidhu B, et al. Disparity in implantable cardioverter defibrillator therapy amongst minority South Asians in the United Kingdom. Heart 2020;106:662-7.

4 Johnson AE, Adhikari S, Althouse AD, et al. Persistent sex disparities in implantable cardioverter-defibrillator therapy. Pacing Clin Electrophysiol 2018;41:1150-7. 
5 Hess PL, Hernandez AF, Bhatt DL, et al. Sex and Race/ Ethnicity differences in implantable cardioverterdefibrillator counseling and use among patients hospitalized with heart failure: findings from the get with the Guidelines-Heart failure program. Circulation 2016;134:517-26.
6 Marzec LN, Peterson PN, Bao H, et al. Use of cardiac resynchronization therapy among eligible patients receiving an implantable cardioverter defibrillator: insights from the National cardiovascular data registry implantable cardioverter defibrillator registry. JAMA Cardiol 2017:2:561-5.
7 Banerjee A. Ethnicity and heart failure. In: Camm AJ, Lüscher TF, Maurer G, eds. Chapter 54.6. ESC CardioMed. 3rd edn. Oxford University Press, 2019.

8 Khatibzadeh S, Farzadfar F, Oliver J, et al. Worldwide risk factors for heart failure: a systematic review and pooled analysis. Int J Cardiol 2013;168:1186-94. 